\title{
On the specific heat of a fermionic atomic cloud in the unitary regime
}

\author{
Aurel Bulgac \\ Department of Physics, University of Washington, Seattle, WA 98195-1560, USA
}

(Dated: January 27, 2019)

\begin{abstract}
In the unitary regime, when the scattering amplitude greatly exceeds in magnitude the average inter-particle separation, and below the critical temperature thermal properties of an atomic fermionic cloud are governed by the collective modes, specifically the Bogoliubov-Anderson sound modes. The specific heat of an atomic cloud in a elongated trap in particular has a rather compex temperature dependence, which changes from an exponential behavior at very low temperatures $\left(T \ll \hbar \omega_{\|}\right)$, to $\propto T$ for $\hbar \omega_{\|} \ll T \ll \hbar \omega_{\perp}$ and then continuosly to $\propto T^{4}$ at temperatures just below the critical temperature, when the surface modes play a dominant role. Only the low $\left(\hbar \omega_{\|} \ll T \ll \hbar \omega_{\perp}\right)$ and high $\left(\hbar \omega_{\perp} \ll T<T_{c}\right)$ temperature power laws are well defined. For the intermediate temperatures one can introduce at most a gradually increasing with temperature exponent.

PACS numbers: 03.75. Ss
\end{abstract}

Dilute atomic Fermi gases [1], and especially dilute gases interacting with large scattering lengths are under intense scrutiny both experimentally $2,3,4$ and theoretically [5, 6, 7, 8, 9, 10, 11, 12, 13, 14, 15] (these lists, especially the list of theoretical contributions, is far from being exhaustive or, maybe, even representative). While quite a number of properties of these systems have been clarified, the full and exact picture still awaits to be uncovered. What was not appreciated so far is the fact that from the information inferred so far, both theoretically and experimentally, one can draw a number of unambiguous conclusions concerning the thermal properties of these systems. It is widely accepted that the equation of state at zero temperature is known theoretically with an accuracy of a few percent [6, 7, []]. This equation of state allows us also to derive the spectrum of low lying collective excitations of such systems 9 , 10, 11, 12, namely the sound waves, and one can safely assume that experimentally 3] the basic properties of these collective modes agree with theory. I shall show here how this information can be profitably used in order to extract information about the thermal properties of these systems at temperatures below the critical temperature for transition into a normal state. I shall also show how the geometry of the these clouds determines in a somewhat unexpected manner the specific heat of these clouds. In the first part of this work I shall discuss briefly some of the properties of the infinite homogeneous systems and I shall turn to the discussion of clouds in harmonic traps in the second part. Various aspects of the thermal properties of fermionic clouds have been considered by others before [13, 14, 15], however, as far as I am aware of, the role of the collective excitations below the pairing gap has not been discussed previously in this context.

In an infinite Fermi system with number density $n=k_{F}^{3} / 3 \pi^{2}$, in the unitary regime, the speed of the
Bogoliubov-Anderson sound mode is given by 12 ]

$$
c^{2}=\frac{\xi_{s} v_{F}^{2}}{3}\left[1-\frac{2 \zeta_{s}}{5 \xi_{s} k_{F} a}+\mathcal{O}\left(\frac{1}{\left(k_{F} a\right)^{2}}\right)\right]
$$

where $v_{F}=\hbar k_{F} / m$ and $k_{F}$ are the Fermi velocity and wave vector respectively and $a$ is the scattering length. The Bogoliubov-Anderson sound waves have the expected linear dispersion law with momentum $\hbar k$, namely $\omega_{s}=c k$. The dimensionless parameters $\xi_{s} \approx 0.44$ and $\zeta_{s} \approx 1$ have been determined recently [ 6,6$],[]$ ]. At low temperatures one can expect that only two types of elementary excitations exist, the boson-like BogoliubovAnderson phonons and the fermion-like gapped Bogoliubov quasi-particles. One can estimate their contribution to the total energy $E(T)$ by assuming that at $T=0$ the system is a Fermi superfluid with a ground state energy and a pairing gap determined in Ref. 6]. One thus obtains [16], see Ref. 17] for derivations of such formulas:

$$
\begin{aligned}
E_{s}(T) & =\frac{3}{5} \varepsilon_{F} N\left[\xi_{s}+\frac{\sqrt{3} \pi^{4}}{16 \xi_{s}^{3 / 2}}\left(\frac{T}{\varepsilon_{F}}\right)^{4}\right. \\
& \left.+\frac{5}{2} \sqrt{\frac{2 \pi \Delta^{3} T}{\varepsilon_{F}^{4}}} \exp \left(-\frac{\Delta}{T}\right)\right],
\end{aligned}
$$

where $\varepsilon_{F}=\hbar^{2} k_{F}^{2} / 2 m, N$ is the total number of particles and the temperature is on the energy scale $\left(k_{B}=1\right)$ and $\Delta$ is the pairing gap at $T=0$. As illustrated in Fig. 1 of Ref. 16 at $T \approx 0.2 \varepsilon_{F}$ the two contributions are essentially equal (each of the last two terms inside the square brackets $\approx 0.06$ ). Off the resonance (when $1 / k_{F} a \neq 0$ ), one has to include corrections similar to those in Eq. (1). Only the (mean-field) exponentially suppressed contribution, due to the breaking of the Cooper pairs, and has been considered previously [14, 15]. Above the critical temperature, such a system behaves as a normal Fermi 
system and its energy can be estimated with the usual textbook formula

$$
E_{n}(T)=\frac{3}{5} \varepsilon_{F} N\left[\xi_{n}+\frac{5 \pi^{2}}{12}\left(\frac{T}{\varepsilon_{F}}\right)^{2}\right],
$$

where $\xi_{n} \approx 0.59$ can be estimated from the condensation energy or be computed directly [6, 18]. Using these two expressions, Eqs. (2) and (3), one can show that the corresponding free energies cross at a temperature $T_{\text {cross }} \approx 0.2 \varepsilon_{F}$. This fact would suggest that the unknown so far critical temperature of a such a system is around $0.2 \varepsilon_{F}$. This estimate is noticeably lower than what a mean-field or BCS-like treatment would suggest in the weak coupling limit, namely $T_{c} \approx 0.5 \varepsilon_{F}[5,[19]$. Notice that even in the weak coupling limit the BCS theory overestimates of the critical temperature 20], and the magnitude of this correction seems to be the same in the strong coupling limit [7]. The above estimate for the critical temperature is rather close to a recent experimental claim [4]. The theoretical mean-field analysis performed in conjunction with this experiment 15], did not consider the role played by the collective modes in the thermal properties for $T<T_{c}$. As it will be shown below, in a trap the thermal properties of a fermion cloud are modified in a qualitative manner and are expected to show the presence of several different regimes.

In typical experiments with cold fermionic atoms the trap is largely harmonic, but it also has a very elongated shape. Since $\hbar \omega_{\|} \ll \hbar \omega_{\perp} \ll \varepsilon_{F}$, the shape of the atomic cloud is that of a very long cigar. The spectrum of the collective oscillations of such a superfluid systems in a spherical trap have been evaluated in Refs. [9]

$$
\Omega_{n l}=\omega \sqrt{\frac{4}{3} n(n+l+2)+l},
$$

where $\omega$ is the frequency of the trap and $n$ and $l$ are the radial quantum number and $l$ is the corresponding angular momentum. One notices that even though the spectrum is distinctly different from a spherical harmonic spectrum, nevertheless it is quite close to one (up to the notorious scale factor $1 / \sqrt{3}$ ), namely

$$
\Omega_{n l} \approx \frac{\omega}{\sqrt{3}}\left(2 n+l+\frac{3}{2}\right) .
$$

This behaviour is similar to the change in dispersion from $\omega=v_{F} k$ for a homogeneous noninteracting Fermi gas to $\omega=v_{F} k / \sqrt{3}$ for a homogeneous Fermi superfluid. Since I shall be interested in global properties of these systems, which depend on the propperties of the entire spectrum of these oscillations, the differences between the exact and approximate spectra, naively, are not expected to change the qualitative picture. For temperatures $T \ll$ $T_{c}=\mathcal{O}(\Delta)=\mathcal{O}\left(\varepsilon_{F}\right)$ only such collective oscillations can be excited with a noticeable probability. The energy of an atomic fermionic elongated cloud is then clearly given by the formula

$$
\begin{gathered}
E_{s}(T)=E(0)+\sum_{n_{x}, n_{y}, n_{z}}^{\prime} \frac{\hbar \Omega\left(n_{x}, n_{y}, n_{z}\right)}{\exp \left[\beta \hbar \Omega\left(n_{x}, n_{y}, n_{z}\right)\right]-1},(6) \\
\Omega\left(n_{x}, n_{y}, n_{z}\right)=\frac{1}{\sqrt{3}}\left[\omega_{\perp}\left(n_{x}+n_{y}\right)+n_{z} \omega_{\|}\right],
\end{gathered}
$$

where $\beta=1 / T$ and the prime superscript is a reminder that only terms with $n_{x}+n_{y}+n_{z}>0$ are to be included in the sum. Since the longitudinal $\omega_{\|}$and radial $\omega_{\perp}$ frequencies differ typically by a factor close to a hundred, one can distinguish several temperature regimes. For very low temperatures, when $T \ll \hbar \omega_{\|}$, the sum in the above formula is exponentially small in comparison with $\hbar \omega_{\|}$and the cloud is essentially in the ground state. The second regime is for temperatures in the interval $\hbar \omega_{\|} \ll T \ll \hbar \omega_{\perp}$, when one can easily show that

$$
E_{s}(T) \approx E(0)+\frac{\sqrt{3} \pi^{2}}{6} \frac{T^{2}}{\hbar \omega_{\|}} .
$$

For temperatures in the interval $\hbar \omega_{\perp} \ll T \ll T_{c}$ one can show that the total energy is essentially quartic in temperature, namely

$$
E_{s}(T) \approx E(0)+\alpha \frac{T^{4}}{\hbar^{3} \omega_{\|} \omega_{\perp}^{2}},
$$

where the constant $\alpha$ can be estimated as the integral

$$
\begin{aligned}
& \alpha=3^{3 / 2} \int_{0}^{\infty} d x \int_{0}^{\infty} d y \int_{0}^{\infty} d z \frac{x+y+z}{\exp (x+y+z)-1} \\
& =\frac{3^{3 / 2} \pi^{4}}{30}=16.87 .
\end{aligned}
$$

Above $T_{c}$ one expects a normal Fermi liquid behavior, with an essentially $T^{2}$ dependence. Naturally, in this rough picture the detailed critical behavior at and around the critical point is neglected. The fact that the energy as a function of temperature can change its character is known in anisotropic solids for a long time [22], however it was not appreciated yet in the context of fermionic atomic clouds in anisotropic traps so far. It is important to realize that the geometric shape of the could alone can lead to this somewhat unexpected behavior of $E(T)$, and of the specific heat as well, and that no phase transition happens really. The transition from the regime corresponding to Eq. (8) to the one corresponding to Eq. (9) is not abrupt, but occurs over a rather long temperature interval, and can be easily described with a temperature dependent exponent.

The approximate spectrum in Eq. (5) does not reproduce correctly the dispersion of the surface modes, namely $\Omega_{\text {surf }} \approx \omega \sqrt{(4 n+3) l / 3}$ (for $n \ll l$ ) and, as it will shown below, their role in the thermal properties of a cloud is somewhat unexpected. One can estimate 
$E(T)$ by evaluating the sum over the quantum numbers $(n, l, m)$. For temperatures $T \gg \Omega$ one then obtains

$$
\begin{aligned}
& E_{s}(T)=E(0)+\sum_{n, l}^{\prime} \frac{(2 l+1) \hbar \Omega_{n l}}{\exp \left(\beta \hbar \Omega_{n l}\right)-1} \\
& \approx \frac{\xi_{s} \hbar \omega(3 N)^{4 / 3}}{4}+142 \frac{T^{5}}{\hbar^{4} \omega^{4}} .
\end{aligned}
$$

Both the temperature exponent and the prefactor have been obtained numerically. One can evaluate this expression also by replacing the sum over $l$ with an integral, namely

$$
\begin{aligned}
& E_{s}(T) \approx E(0)+\sum_{n=0}^{\infty} 2 \hbar \omega \sqrt{1+\frac{4 n}{3}} \\
& \times \int d l l^{3 / 2} \exp \left(-\frac{\hbar \omega l^{1 / 2}}{T} \sqrt{1+\frac{4 n}{3}}\right) \\
& \approx 0.25 \xi_{s}^{1 / 2} \hbar \omega(3 N)^{4 / 3}+140 \frac{T^{5}}{\hbar^{4} \omega^{4}} .
\end{aligned}
$$

Naturally, there are sub-leading corrections to these estimates, notably corrections of order $\mathcal{O}\left(T^{4}\right)$. It is desirable to extend this formula to the case of an axially deformed trap. Unfortunately a simple analytical formula for the collective modes is not available in this case 23]. One can use the fact that the (local) frequency $\Omega_{\text {surf }}(S)$ of the surface modes with wave vector $k$ can be determined rather accurately from the classical formula

$$
\Omega_{\text {surf }}^{2}(S)=k \frac{F(S)}{m},
$$

where $F(S)=|\nabla U(\mathbf{r})|_{S}$ is the force acting on a particle at the surface of the cloud, see Refs. 24] for a detailed discussion. In the above formula the argument $S$ stands for a particular point on the cloud surface. Using an axially symmetric harmonic trapping potential $U(\mathbf{r})=$ $m \omega^{2}\left(x^{2}+y^{2}+\lambda^{2} z^{2}\right) / 2$ and the semiclassical formula for the contribution of the surface modes to the total energy

$$
\begin{aligned}
& E_{s}(T) \approx E(0)+\int \frac{d S d^{2} k}{(2 \pi)^{2}} \frac{\hbar \Omega_{\text {surf }}(S)}{\exp \left[\beta \hbar \Omega_{\text {surf }}(S)\right]-1} \\
& \approx 0.25 \xi_{s}^{1 / 2} \hbar \omega(3 N)^{4 / 3}+\frac{96 T^{5}}{\hbar^{4} \omega^{4}} \frac{\arctan \sqrt{\lambda^{2}-1}}{\lambda \sqrt{\lambda^{2}-1}} .
\end{aligned}
$$

Here $\int d S$ stands for the integral over the surface of the cloud. In the case of a spherical trap $(\lambda=1)$ one recovers the previous formula, see Eq. (12), if one includes only the modes with $n=0$. One can thus conclude that the effect of the effect of the deformation of the trapping potential can be encapsulated in a simple shape factor. Since most experiments are performed in cigar-like traps $(\lambda \ll 1)$, the role of the surface modes is even larger $(\propto 1 / \lambda)$, when compared to spherical traps.

It is useful to obtain an estimate of the maximum temperature up to which this formula is expected to be valid.
One assumption is that $T<\Delta(T)$, which is likely to hold up to temperatures $T \approx T_{c} / 2$. A reasonable estimate of the important angular momenta in the above integral is

$$
l \approx\left(\frac{T}{\hbar \omega}\right)^{2}<l_{\max } \approx(24 N)^{1 / 3}
$$

where $l_{\max }$ is a rough estimate of the largest singleparticle angular momentum of a fermion in the ground state. Using this estimate one obtains for the total energy the following rough estimate

$$
E_{s}(T)<\frac{\xi_{s}^{1 / 2} \hbar \omega(3 N)^{4 / 3}}{4}+140 \hbar \omega(24 N)^{5 / 6} .
$$

Perhaps the thermal energy stored in the surface modes is grossly overestimated. At high, but not yet determined, temperatures the surface will "melt" and the $T^{5}$ regime will be replaced by a normal Fermi liquid behavior at some point. While a good case could be made that the exact numerical factor in front of the second term in the above formula is too large, the dependence on the particle number is probably correct. One can easily estimate the free energy as well and obtain that

$$
\begin{aligned}
F_{s}(T) & =\frac{\xi_{s}^{1 / 2} \hbar \omega(3 N)^{4 / 3}}{4}-35 \frac{T^{5}}{\hbar^{4} \omega^{4}} \\
& >\frac{\xi_{s}^{1 / 2} \hbar \omega(3 N)^{4 / 3}}{4}-35 \hbar \omega(24 N)^{5 / 6}
\end{aligned}
$$

which would have to be contrasted with the free energy of the normal phase

$$
F_{n}(T)=\frac{\xi_{n}^{1 / 2} \hbar \omega(3 N)^{4 / 3}}{4}-\frac{\pi^{2}(24 N)^{2 / 3} T^{2}}{24 \xi_{n}^{1 / 2} \hbar \omega} .
$$

This estimate of the free energy of the normal phase implies that no collective modes exist. Since the interaction between fermions is attractive, the Landau's zero sound mode is unstable. There is still the question of whether the first sound mode can exist in such a system at relatively low temperatures in the normal phase. If the first sound modes would be stable, then their contribution to the free energy would be very similar to the contribution of the Bogoliubov-Anderson sound modes to the free energy of the superfluid phase. So far neither theoretical nor experimental evidence of a first sound mode in such systems in the unitary regime exist. This fact should be not construed, however, as evidence of its non-existence.

If no first sound modes exist, then the inescapable conclusion emerges that the free energy of the superfluid phase is likely well below the free energy of the normal phase in a trap for a temperature range much larger than in the bulk. That would mean that an atomic fermionic cloud in the unitary regime remains superfluid at temperatures larger than the bulk critical temperatures. In the above analysis I have obviously neglected the contribution due to thermally broken Cooper pairs. This 
contribution, which has been considered previously by others, was not the subject of this work.

In conclusion, the thermal properties of fermionic atomic gases, especially in the unitary regime, when the scattering length greatly exceeds in magnitude the average inter-particle separation, are dominated by the thermal excitation of the Bogoliubov-Anderson sound modes, both in the bulk and in traps. In traps, the specific heat of such a system, at temperatures below the critical transition to a normal phase, has a rather complicated temperature dependence, ranging from exponentially damped at very low $T$ 's to various power laws. At temperatures higher than the axial frequency of an elongated trap the specific heat has a linear in $T$ regime, due to the excitation of predominantly axial sound modes. At higher temperatures, close but below the critical temperature, this regime is replaced with a regime in which $C \propto T^{4}$, when the excitation of pure surface modes dominate the thermal properties of atomic clouds in traps. This behavior is obviously absent in the bulk and is characteristic of finite systems only. A similar behavior of the specific heat is also expected in the case of an atomic Bose gas in a trap, since the spectrum of surface mode is similar [21].

A number of conversations with my colleagues G.F. Bertsch and D.J. Thouless have been very helpful for clarifying some of my initial ideas. I thank J.E. Drut for correcting a slip, P. Magierski for reading the draft and D.T. Son for discussions. This work was supported in part by the Department of Energy under grant DEFG03-97ER41014.

[1] B. DeMarco and D.S. Jin, Science, 285, 1703 (1999).

[2] K.M. O'Hara et al., Phys. Rev. Lett. 82, 4204 (1999); K.M. O'Hara, et al., Science, 298, 2179 (2002); M.E. Gehm, et al., Phys. Rev. A 68, 011401 (2003); T. Bourdel, et al., Phys. Rev. Lett. 91, 020402 (2003); C. A. Regal, et al., Nature 424, 47 (2003); K.E. Strecker, et al., Phys. Rev. Lett. 91, 080406 (2003); J. Cubizolles, et al., Phys. Rev. Lett. 91, 240401 (2003); S. Jochim, et al., Phys. Rev. Lett. 91, 240402 (2003); K. Dieckmann, et al., Phys. Rev. Lett. 89, 203201 (2002); C.A. Regal, et al., Phys. Rev. Lett. 92, 083201 (2004); M. Greiner, et al., Nature 426, 537 (2003); M.W. Zwierlein, et al., Phys. Rev. Lett. 91, 250401 (2003); S. Jochim, et al., Science 302, 2101 (2003); M. Bartenstein, et al., Phys. Rev. Lett. 92, 120401 (2004); T. Bourdel et al., cond-mat/0403091 C.A. Regal, et al., Phys. Rev. Lett. 92, 040403 (2004); M.W. Zwierlein, et al., Phys. Rev. Lett. 92, 120403 (2004); C. Chin et al., Science 305,1128 (2004).

[3] J. Kinast et al., Phys. Rev. Lett. 92, 150402 (2004); M.
Bartenstein, et al., Phys. Rev. Lett. 92, 203201(2004).

[4] J. Kinast et al., cond-mat/0409283 see also [15].

[5] E. Timmermans, et al., Phys. Lett. A 285, 228 (2001); M. Holland, et al., Phys. Rev. Lett. 87, 120406 (2001); Y. Ohashi and A. Griffin, Phys. Rev. Lett. 89, 130402 (2002) and references therein.

[6] J. Carlson, et al., Phys. Rev. Lett. 91, 050401 (2003).

[7] S.-Y. Chang et al., Phys. Rev. A 70, 043602 (2004).

[8] G. Astrakharchik et al., Phys. Rev. Lett. 93, 200404 (2004).

[9] M. Amoruso et al., Eur. Phys. J D 7, 441 (1999); G.M. Bruun and C.W. Clark, Phys. Rev. Lett. 83, 5415 (1999); M.A. Baranov and D.S. Petrov, Phys. Rev. A 62, 04601(R) (2000).

[10] H. Heiselberg, Phys. Rev. Lett. 93, 250402 (2004); C. Menotti et al., Phys. Rev. Lett. 89, 250402 (2002); L. Vichi and S. Stringari, Phys. Rev. A 60, 4734 (1999); M. Cozzini and S. Stringari, Phys. Rev. Lett. 91, 070401 (2003); H. Hu et al., Phys. Rev. Lett. 93, 190403 (2004); M. Manini and L. Salasnich, cond-mat/0407039 Y.E. Kim and A.L. Zubarev, Phys. Lett. A 327, 2004); Phys. Rev. A 70, 033612 (2004); R. Combescot and X. Leynoras, Phys. Rev. Lett. 93, 138901 (2004); Europhys. Lett. 68, 762 (2004).

[11] S. Stringari, Europhys. Lett. 65, 749 (2004).

[12] A. Bulgac and G.F. Bertsch, Phys. Rev. Lett. 94, 070401 (2005).

[13] T.-L. Ho, Phys. Rev. Lett. 92, 090402 (2004).

[14] H. Heiselberg, cond-mat/0409077

[15] Q. Chen et al., cond-mat/0411090 this e-print has been combined with the e-print cond-mat/0409283 [4] and published as joint paper, J. Kinast et al., Science 307, 1296 (2005).

[16] A. Bulgac et al., cond-mat/0505374

[17] A.L. Fetter and J.D. Walecka, Quantum Theory of ManyParticle Systems, McGraw-Hill, San Francisco 1971.

[18] J. Carlson et al., Phys. Rev. C 68, 025802 (2003).

[19] D.R. Eagles, Phys. Rev. 186, 456 (1969); A.J. Leggett, in Modern Trends in the Theory of Condensed Matter, eds. A. Pekalski and R. Przystawa, Springer-Verlag, Berlin, 1980; J. Phys. (Paris) Colloq. 41, C7-19 (1980); P. Nozières and S. Schmitt-Rink, J. Low Temp. Phys. 59, 195 (1985); C.A.R. Sá de Mello et al., Phys. Rev. Lett. 71, 3202 (1993); M. Randeria, in Bose-Einstein Condensation, eds. A. Griffin, D.W. Snoke and S. Stringari, Cambridge University Press (1995), pp 355-392.

[20] L.P. Gorkov and T.K. Melik-Barkhudarov, Sov. Phys. JETP 13, 1018 (1961); H. Heiselberg, et al., Phys. Rev. Lett. 85, 2418 (2000).

[21] A. Griffin et al., Phys. Rev. Lett. 78, 1838 (1997).

[22] E.M. Lishitz and L.P. Pitaevskii, Statistical Physics, part 1, Pergamon Press, Oxford, 1980.

[23] A. Csordas and R. Graham, Phys. Rev. A 63, 013606 (2001); ibid Phys. Rev. A 64, 013619 (2001).

[24] C.J. Pethick and H. Smith, Bose-Einstein Condensation in Dilute Gases, Cambridge University Press, Cambridge, 2002; L.P. Pitaevskii and S. Stringari, Bose Einstein Condensation, Oxford University Press, Oxford, 2003. 